\title{
Characteristics of Residual Symptoms in Korean Patients with Major Depressive Disorder: A Validation Study for the Korean Version of Depression Residual Symptom Scale
}

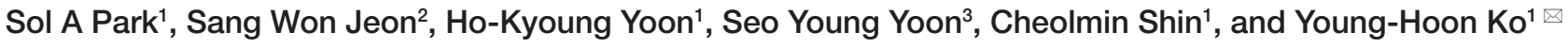 \\ ${ }^{1}$ Department of Psychiatry, Korea University College of Medicine, Ansan Hospital, Ansan, Republic of Korea \\ ${ }^{2}$ Department of Psychiatry, Sungkyunkwan University School of Medicine, Kangbuk Samsung Hospital, Seoul, Republic of Korea \\ ${ }^{3}$ Department of Psychiatry, Catholic University of Daegu School of Medicine, Daegu, Republic of Korea
}

Objective Residual symptoms of depression are related to more severe and chronic course of functional impairment with higher risk of relapse. The objective of this study was to validate, and determine psychometric properties of the Korean version of Depression Residual Symptom Scale (KDRSS).

Methods A total of 203 outpatients with recent episode of major depression based on DSM-IV criteria were enrolled in this study. They had been treated with antidepressants and assessed by KDRSS, Hamilton Depression Rating Scale-24 (HDRS-24), and Montgomery-Åsberg Depression Rating Scale (MARDS). The validity and reliability of KDRSS were assessed, including internal consistency reliability, concurrent validity, temporal stability, factorial validity, and discriminative validity.

Results Internal consistency (Cronbach's alpha=0.961), concurrent validity (MADRS: $r=0.731, p<0.01$, HDRS-24: $r=0.663$, $p<0.01$ ), and temporal stability $(\mathrm{r}=0.726, \mathrm{p}<0.01)$ of KDRSS were all excellent. KDRSS showed good discriminative validity based on MARDS. KDRSS consisted of one-factor structure accounting for $63.8 \%$ of total variance. All subjects except two in full remission group had one or more residual symptoms. In 7 subscales of KDRSS consisting of similar items respectively, 'lack of energy' was the most commonly reported, followed by 'increased emotionalism' in this group.

Conclusion KDRSS is a useful and sensitive instrument for measuring residual depressive symptoms. Since some depressive symptoms including 'lack of energy' and 'increased emotionalism' in patients with full remission might be persistent during psychiatric intervention, these symptoms need to be focused on in clinical practice.

Psychiatry Investig 2018;15(2):178-185

Key Words Major depressive disorder, Remission, Depression residual symptom scale.

\section{INTRODUCTION}

Full remission of a depressive episode with functional recovery is the goal of first-line treatment for major depressive disorder. However, approximately one-third of patients are non-responders to treatment, although one-third of patients do show response, and another one-third of patients reach complete remission in clinical settings. ${ }^{1}$ In addition, patients with

Received: May 6, 2017 Revised: June 23, 2017

Accepted: July 13, 2017 Available online: October 12, 2017

$\triangle$ Correspondence: Young-Hoon Ko, MD, PhD

Department of Psychiatry, College of Medicine, Korea University, Ansan Hospital, 123 Jeokgeum-ro, Danwon-gu, Ansan 15355, Republic of Korea

Tel: +82-31-412-5140, Fax: +82-31-412-5144,

E-mail: koyh@korea.ac.kr

(a) This is an Open Access article distributed under the terms of the Creative Commons Attribution Non-Commercial License (http://creativecommons.org/licenses/bync/4.0) which permits unrestricted non-commercial use, distribution, and reproduction in any medium, provided the original work is properly cited. full remission might still suffer from depression-related symptoms (called residual symptoms) with various intensities. Residual symptoms of a major depressive episode (MDE) have higher probability to cause subsequent depressive episode with chronic course of depression in a majority of follow-up studies.

The most common residual depressive symptoms include sleep disturbance, sadness, diminished concentration, and psychomotor disturbance. ${ }^{2}$ Patients with residual symptoms exhibit more significant psychosocial and socio-economic impairments in their life. ${ }^{3}$ Presence of residual symptoms at recovery from a MDE is related to higher rates of relapse and recurrence and more severe and longer duration of depressive episode over a long follow-up period. ${ }^{4-7}$ Moreover, having residual symptoms is considered a better predictor of early relapse and shorter time to a subsequent episode than past history of depression..$^{8-11}$ 
Therefore, evaluation of residual symptoms is clinically important because the prognosis and outcome of treatment in depression may depend on the severity of residual symptoms and the degree of remission. ${ }^{3,6,12}$ Hamilton Depression Rating Scale (HDRS) and Montgomery-Åsberg Depression Rating Scale (MADRS) have been widely used to assess the severity of acute depressive symptoms. However, these scales are not originally designed to measure or evaluate residual symptoms of depression. Accurate measurement of residual symptoms in depression can help clinicians make therapeutic decisions to decrease relapse risk and monitor a patient's treatment course. ${ }^{13}$

Depression Residual Symptom Scale (DRSS) was developed in Europe to assess residual symptoms in patients with a full remission of MDE.$^{14}$ DRSS mainly reflects patient's self-perception of psychological residual symptoms such as subjective symptoms of vulnerability, loss of self-confidence, and lack of return to usual self that are important for determining remission. ${ }^{14,15}$ The objective of this study was to validate Korean version of Depression Residual Symptom Scale (KDRSS) and evaluate the usefulness of KDRSS for the identification of residual symptoms in patients with depression. In addition, the characteristics of residual symptoms in Korean patients with full remission in depression were explored.

\section{METHODS}

\section{Study design and subjects}

This was a prospective and observational study conducted in a single center by three psychiatrists for one year. Study participants were consecutive outpatients with current MDE based on the Diagnostic and Statistical Manual of Mental disorders (fourth edition diagnostic criteria) who had been treated with antidepressants for over one month. Although DRSS was developed to evaluate the residual symptoms in fully remitted patients with depression, other patients including remitters were also recruited to find whether KDRSS score reflected the state and severity of depression. Full remission of a major depressive episode was defined when HDRS-24 score was less than $10 .^{16}$

The age of study subjects was 18 to 65 years old. They were able to read and write Korean. To minimize confounding, the following exclusion criteria were established: 1) a history of substance dependence or abuse; 2) psychotic symptoms, catatonic features, or severe psychomotor retardation; 3) pregnant or breast feeding patients; and 4) severe medical or surgical disorder which developed in a month before recruitment. This study was approved by Korean University Ansan Hospital Institutional Review Board (AS11094). Written informed consent was obtained from all subjects prior to study enrollment.

\section{Linguistic adaptation for the original version of DRSS}

DRSS consists of 25 items with an affirmative sentence comparing post-depression status with pre-depression status using the following seven subscales: sadness and anhedonia, lack of energy, psychomotor retardation, anxiety, subjective feelings of vulnerability, loss of internal reference points, and increased emotionalism. ${ }^{14}$ When calculating DRSS score, 'Wrong, 'Slightly true, 'Moderately true, and 'Completely true' were scored as $0,1,2$, and 3 points, respectively. The Korean version of DRSS was developed using a forward-backward translation process. In the translation process, these items were firstly translated into Korean by one psychiatrist whose native language was Korean. Two other bilingual Korean professionals then performed back translation blindly. Next, the back-translated items in English were compared to the original ones. If a back-translated item did not agree with the original one, revision and back translation were performed repeatedly until these items were consistent with the intent of the original instrument.

\section{Psychometric properties of KDRSS}

After subjects were recruited, patients completed KDRSS and psychometric instruments including a questionnaire on socio-demographic factors. A psychiatrist evaluated MADRS and HDRS-24. The two have been validated and widely used in Korea. To examine the stability of KDRSS scale, patients completed the KDRSS at 4 weeks after baseline assessment.

The MADRS is a 10-item depression rating scale widely used to measure severity of depression in clinical practice. It is sensitive to changes during antidepressant treatment. ${ }^{17}$ It provides more internally consistent and more precise estimates of depression severity than HDRS or other rating scales. ${ }^{18-20}$ Since most subjects had ongoing treatment, discriminative validity and construct validity of KDRSS were explored using MADRS with more focus toward psychological and subjective aspects of depression. ${ }^{21}$ HDRS is one of the most widely used clinician-rated scales in depression. It has been accepted as a valid tool for depression severity assessment. ${ }^{22}$ HDRS provides more representation to anxiety, somatic symptoms, and vegetative symptoms compared to MADRS which usually accounts for residual depressive symptoms in clinical practice. To examine distinguishable characteristics of residual symptoms in KDRSS, participants were divided into two groups based on HDRS-24 scores: full remission (FR) group and nonfull remission (NFR) group.

\section{Data analyses}

Data were reported as means \pm standard deviation (SD) or percentages where appropriate. Independent $\mathrm{t}$-test was used to compare continuous variables while chi-square test was used to compare categorical variables. Internal consistency of KDRSS 
was assessed with Cronbach's alpha and corrected item-total correlation. Pearson's correlation analysis was performed to examine construct validity of KDRSS against MARDS. The stability of KDRSS over 4 weeks was analyzed using Pearson's correlation coefficient. To evaluate factorial validity, principal component factor analysis with varimax rotation was performed. Repeated-measure analysis of variance (ANOVA) with Bonferroni correction was used to find differences in scores of KDRSS over 4 weeks between FR and NFR groups. Post hoc t-test in each group was performed if group-by-time interaction or group difference was significant. Two-tailed p-value of less than 0.05 was considered statistically significant. Data were analyzed using PASW Statistics 18 statistical software (IBM Corp., Armonk, NY, USA).

\section{RESULTS}

\section{Demographic characteristics of subjects}

A total of 203 subjects (61 males and 142 females) were included in the study. Their mean age was $45.6 \pm 13.1$ years. Among these 203 subjects, 164 ( 45 men and 119 women) completed the 4-week follow-up visit. The mean baseline score of MARDS was $14.5 \pm 10.4$ and that of HDRS-24 was $13.5 \pm 9.5$. A total of 86 (42.4\%) subjects showed full remission while 117 (57.6\%) subjects failed to show full remission. The female to male ratio in the FR group was similar to that of the NFR group. However, the average age was higher in the FR group while the education level was higher in the NFR group. There was no significant difference in illness duration between the two groups. Clinical characteristics of subjects in this study are summarized in Table 1.

Table 1. Demographic and clinical characteristics of subjects $(\mathrm{N}=203)$

\begin{tabular}{lccc}
\hline Characteristics & FR group & NFR group & p-value \\
\hline Subjects, N (\%) & $86(42.4)$ & $117(57.6)$ & \\
Male & $25(29.1)$ & $36(30.8)$ & 0.794 \\
Female & $61(70.9)$ & $81(69.2)$ & 0.794 \\
Age, mean \pm SD (years) & $48.7 \pm 12.5$ & $43.3 \pm 13.1$ & 0.004 \\
Education level, mean \pm SD & $9.9 \pm 4.0$ & $11.3 \pm 3.3$ & 0.015 \\
$\quad$ years) & & & \\
Illness duration, mean $\pm S D$ & $6.2 \pm 6.8$ & $6.4 \pm 5.4$ & 0.849 \\
$\quad$ years) & & & \\
MARDS score, mean $\pm S D$ & $6.0 \pm 4.1$ & $20.7 \pm 9.2$ & $<0.001$ \\
HDRS-24 score, mean $\pm S D$ & $5.0 \pm 2.4$ & $19.7 \pm 7.8$ & $<0.001$ \\
KDRSS score, mean $\pm S D$ & $17.8 \pm 13.1$ & $44.4 \pm 15.2$ & $<0.001$ \\
\hline
\end{tabular}

MARDS: Montgomery-Åsberg Depression Rating Scale, HDRS-24: Hamilton Depression Rating Scale-24, KDRSS: Korean Version of Depression Residual Symptom Scale, SD: standard deviation

\section{Internal consistency reliability, concurrent validity, and temporal stability}

Cronbach's alpha coefficient for KDRSS was calculated to be 0.961 . Correlation coefficients between each item and the total score ranged from 0.272 to 0.776 . Cronbach's alpha for all 25 items, if individual item was deleted, is summarized in Table 2. Item 14 was the only item that increased Cronbach's alpha from 0.961 to 0.967 when it was deleted. It had the lowest corrected item-total correlation. The internal consistency in the FR group was 0.930. It was 0.931 in the NFR group. Corrected item-total correlation of KDRSS ranged from 0.604 to 0.826 except for item 14 assessing emotionalism. It had a low correlation of - 0.249 . Items $13,14,15$ had relatively low correlations (below 0.5) in both FR and NFR group. KDRSS showed statistically significant and positive correlations with MARDS $(\mathrm{r}=0.731, \mathrm{p}<0.01)$. It was temporally stable in 4 weeks $(\mathrm{r}=0.726$, $\mathrm{p}<0.01)$.

\section{Factorial validity}

The Kaiser-Meyer-Olkin measure of sampling adequacy was 0.955 and the p-value of Bartlett's test of sphericity was below 0.001 , indicating that data were appropriate for factor analysis and the presence of common factor. In factor analysis for 25 items in KDRSS, three components accounted for $63.8 \%$ of the total variation. Eigenvalues of the three factors were $13.77,1.13$, and 1.06, respectively. Factor 1 consisted of a total of 17 items accounting for $55.1 \%$ of the total variation. Factor 2 comprised of 7 items accounting for $4.5 \%$ of the total variation. Factor 3 composed of only one item (item 14). It was against the guideline that at least three components were necessary for a significant factor. ${ }^{23}$ Cronbach's alpha coefficient of factor 1 and factor 2 was 0.961 and 0.879 , respectively, suggesting that both factors were adequate. In addition, the ratio between the eigenvalue of the factor 1 and that of factor 2 was 12.19 , exceeding the critical value of 4 to be indicative of unidimensionality. ${ }^{24}$ Therefore, KDRSS consisted of one-factor structure accounting for $63.8 \%$ of the total variation.

\section{Discriminative validity}

To evaluate the discriminative validity of KDRSS, subjects were divided into four severity groups according to MADRS score: 0-6, minimal; 7-19, mild; 20-34, moderate; and $\geq 35$, severe severity. ${ }^{25}$ The KDRSS scores of the four groups from the least to the most severe were $14.5 \pm 10.9,33.3 \pm 16.0,48.7 \pm 13.4$, and $54.9 \pm 13.4$, respectively. When differences in scores of KDRSS were compared, all Turkey post-hoc comparisons showed significant differences among groups $[\mathrm{F}(3,199)=66.6, \mathrm{p}<0.001]$.

\section{Changes in score of KDRSS over 4 weeks}

Repeated-measure ANOVA was used to explore changes in 
Table 2. Reliability with Cronbach's Alpha if item is deleted in FR or NFR group at baseline

\begin{tabular}{|c|c|c|c|}
\hline Items & FR & NFR & Total \\
\hline \multicolumn{4}{|l|}{ Subjective feelings of vulnerability } \\
\hline Item 1: I feel more vulnerable to stress than before & 0.929 & 0.929 & 0.960 \\
\hline Item 4: I feel more vulnerable than before & 0.925 & 0.928 & 0.958 \\
\hline Item 5: Something has broken inside me and is not repaired & 0.926 & 0.927 & 0.958 \\
\hline Item 11: I adapt to changes less easily than before & 0.924 & 0.926 & 0.958 \\
\hline Item 19: I am less confident than before & 0.925 & 0.927 & 0.958 \\
\hline \multicolumn{4}{|l|}{ Psychomotor retardation } \\
\hline Item 2: I have more memory difficulties than before & 0.928 & 0.930 & 0.959 \\
\hline Item 10: I have the feeling of acting and moving more slowly than before & 0.926 & 0.927 & 0.958 \\
\hline Item 21: I have more difficulties to take decisions than before & 0.925 & 0.926 & 0.958 \\
\hline \multicolumn{4}{|l|}{ Lack of energy } \\
\hline Item 3: To start something demands a greater effort than before & 0.926 & 0.928 & 0.959 \\
\hline Item 6: I need to have a more regular life than before & 0.929 & 0.929 & 0.959 \\
\hline Item 7: After an effort, I recover less easily than before & 0.924 & 0.926 & 0.958 \\
\hline Item 13: In the morning, I get up with more difficulties than before & 0.929 & 0.930 & 0.959 \\
\hline \multicolumn{4}{|l|}{ Anxiety } \\
\hline Item 8: I feel my muscles strained more often than before & 0.925 & 0.929 & 0.958 \\
\hline Item 12: I feel anxious more easily than before & 0.924 & 0.926 & 0.958 \\
\hline \multicolumn{4}{|l|}{ Loss of internal reference points } \\
\hline Item 9: I have a sensation of emptiness inside me more often than before & 0.924 & 0.927 & 0.958 \\
\hline Item 15: When I feel sad, I wonder whether depression is starting again & 0.933 & 0.931 & 0.961 \\
\hline Item 24: I feel my life as being less meaningful than before & 0.926 & 0.927 & 0.958 \\
\hline \multicolumn{4}{|l|}{ Increased emotionalism } \\
\hline Item 14: I feel closer to my nearest than before & 0.937 & 0.939 & 0.967 \\
\hline Item 16: I feel emotions more strongly than before & 0.926 & 0.929 & 0.959 \\
\hline Item 23: I am more influenced by the weather than before & 0.927 & 0.930 & 0.960 \\
\hline \multicolumn{4}{|l|}{ Sadness and anhedonia } \\
\hline Item 17: I am less attracted by novelty than before & 0.927 & 0.927 & 0.959 \\
\hline Item 18: I feel joy less easily than before & 0.925 & 0.927 & 0.958 \\
\hline Item 20: I want to see people less than before & 0.927 & 0.927 & 0.958 \\
\hline Item 22: I feel sad more easily than before & 0.926 & 0.927 & 0.958 \\
\hline Item 25: I see the society in a more negative way than before & 0.927 & 0.926 & 0.959 \\
\hline
\end{tabular}

FR: full remission, NFR: non-full remission

score of KDRSS over time and compare differences between FR $(n=68)$ and NFR ( $n=96)$ groups. Figure 1 shows changes in total score and the score of each subscale of KDRSS over 4 weeks between the two groups. Total KDRSS score showed significant group differences $(\mathrm{p}<0.001)$ and group-by-time interactions $(\mathrm{p}=0.006)$ by repeated-measure ANOVA. Posthoc tests showed that the mean total score of the KDRSS after 4 weeks was 17.0 in the FR group, which was not significantly different from that (17.8) at baseline. The mean total score of the KDRSS after 4 weeks in the NFR group was 36.4, which was significantly $(\mathrm{p}<0.001)$ decreased compared to that (44.4) at baseline. While group-by-time interaction was found in subscale of lack of energy ( $\mathrm{p}=0.028)$, anxiety $(\mathrm{p}<0.001)$, and subjective feelings of vulnerability ( $\mathrm{p}=0.023$ ), all subscales showed significant $(\mathrm{p}<0.001)$ group differences over 4 weeks.
Post-hoc analyses with Bonferroni correction revealed that the FR group did not show any significant change in the mean score of each subscale over 4 weeks. However, the NFR group showed significant changes in the mean score of each subscale except 'increased emotionalism' over 4 weeks.

\section{Characteristics of residual symptoms in patients with full-remission}

To evaluate whether KDRSS could explain residual symptoms in patients with depression and find which symptoms were more frequent, the number of full remitters with mildto-severe symptoms in each subscale and the number of them with multiple residual symptoms were explored. Residual symptom was defined if the mean score of each of 7 subscales was 1 or above (mild-to-severe), meaning that patients 

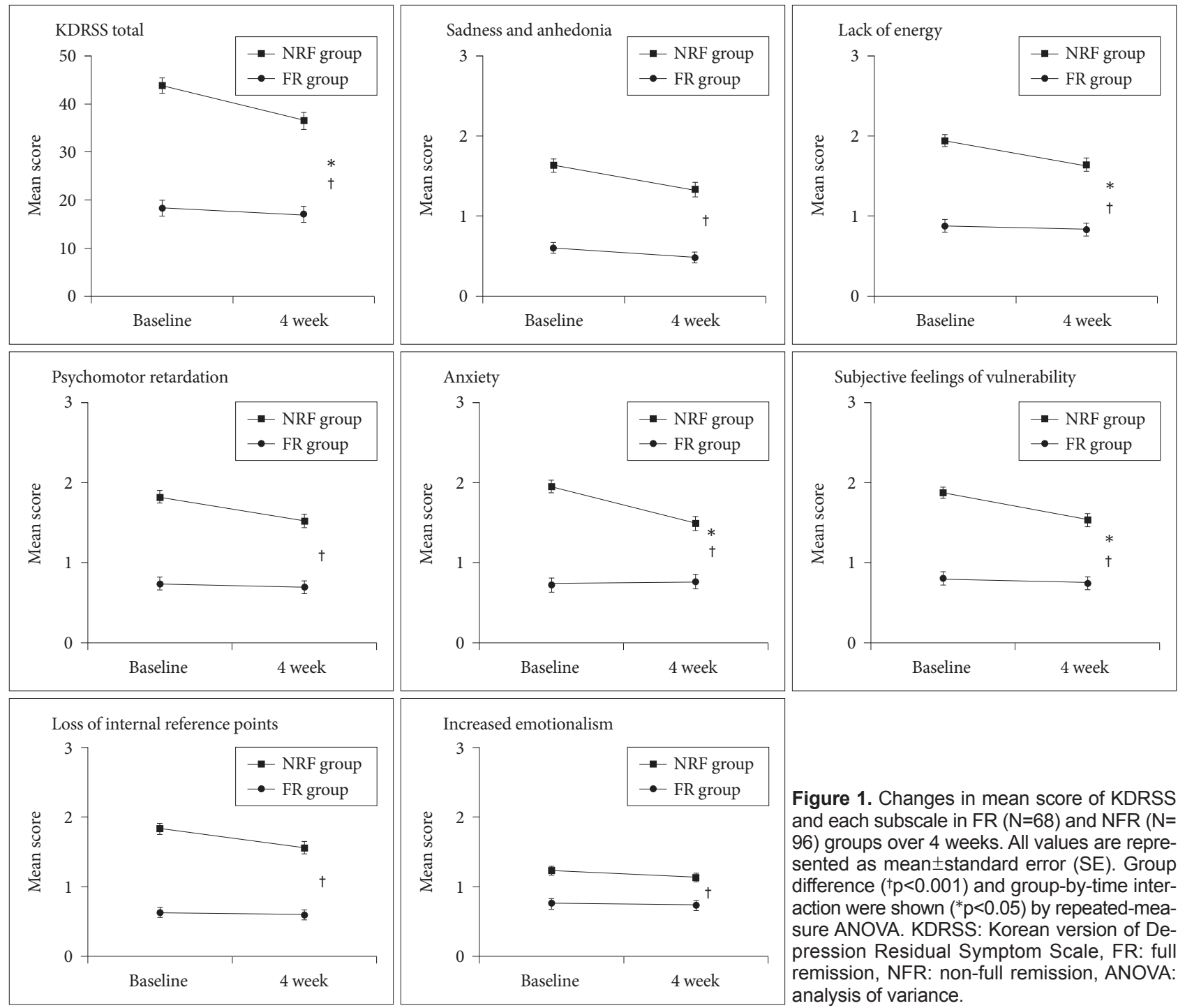

Figure 1. Changes in mean score of KDRSS and each subscale in FR $(\mathrm{N}=68)$ and $\mathrm{NFR}(\mathrm{N}=$ 96) groups over 4 weeks. All values are represented as mean \pm standard error (SE). Group difference $\left({ }^{\dagger} p<0.001\right)$ and group-by-time interaction were shown $\left({ }^{*} p<0.05\right)$ by repeated-measure ANOVA. KDRSS: Korean version of Depression Residual Symptom Scale, FR: full remission, NFR: non-full remission, ANOVA: analysis of variance.

had several similar symptoms in a subscale. The most common residual symptom was lack of energy ( $\mathrm{n}=39,45.4 \%)$, followed by increased emotionalism $(\mathrm{n}=35,40.7 \%)$ and subjective feelings of vulnerability ( $\mathrm{n}=34,39.5 \%$ ) (Figure 2). Sixtytwo subjects $(72.1 \%)$ in the FR group had at least one residual symptom. The mean number of residual symptoms was $2.55 \pm$ 2.38 in each subject in the FR group (Figure 3).

\section{DISCUSSION}

The KDRSS showed good internal consistency, concurrent validity, and temporal stability. In addition, it was found to be a sensitive self-report scale to evaluate psychological residual symptoms of depression which could be easily missed in clinical practice.

Items of KDRSS indicate psychological impact of depression. These items might reflect personality traits of the subject.

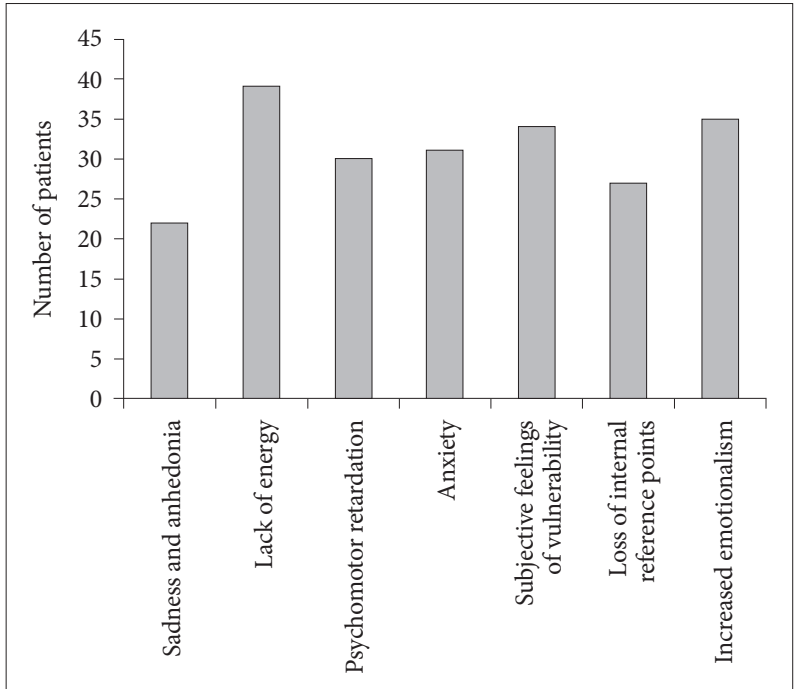

Figure 2. Number of patients with mild-to-severe symptoms of each subscale in the full remission group $(\mathrm{N}=86)$. 


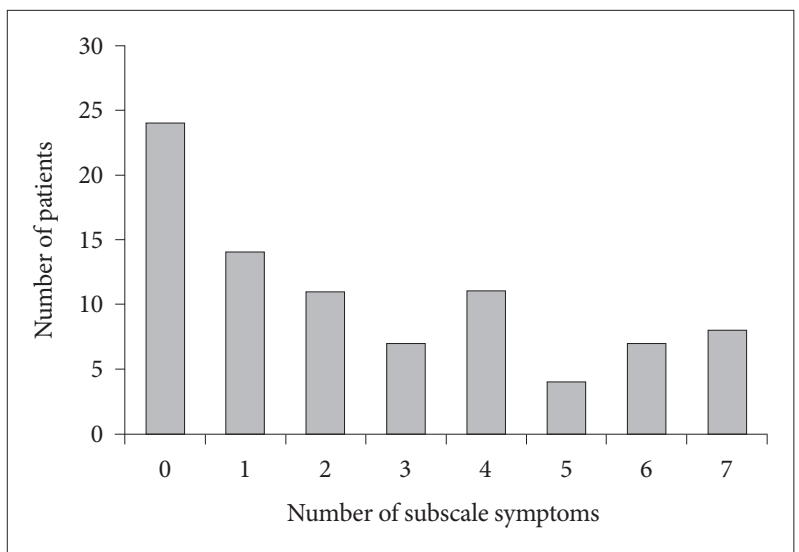

Figure 3. Number of patients with multiple symptoms in the full remission group $(\mathrm{N}=86)$.

To understand whether KDRSS could evaluate the state and severity of depression, patients with NFR were recruited, in addition to those with FR. Repeated-measure ANOVA showed significant group differences and group-by-time interactions of mean total score between NFR and FR patients. Moreover, post-hoc tests revealed that mean total score of KDRSS in the FR group was not significantly changed from baseline to 4 weeks, whereas that in the NFR group was significantly decreased from baseline to 4 weeks. These results indicate that the NFR group with higher KDRSS scores might be more likely to benefit from treatment. Moreover, these results suggest that KDRSS is good for evaluating symptoms of depression, especially residual symptoms in the FR group. These results were also supported by additional analyses using repeatedmeasure ANOVA for subscales of KDRSS.

The subscales of KDRSS showed significant group differences or group-by-time interactions over 4 weeks between FR and NFR groups. FR group had no significant change in scores of each subscale, meaning that residual symptoms were not changed much in the FR group over 4 weeks. Therefore, residual symptoms of depression should be targeted and closely monitored during treatment in full remitters. On the other hand, the NFR group showed significant changes in scores of each subscale except the subscale of increased emotionalism. Therefore, NFR patients with higher scores of KDRSS subscales are more likely to obtain greater benefit from 4 weeks of treatment as mentioned above.

However, these results might be due to confounding effect of 'increased emotionalism' subscale including item 14 . The item 14 showed a negative corrected item-total correlation. The Cronbach's alpha increased the most after it was deleted. Factor analysis showed that item 14 was an isolated factor. In a previous research, although item 14 was found to be one of three items with the lowest corrected item-total correlation coefficients, Bertschy et al. ${ }^{14}$ kept all items in DRSS because
Cronbach's alpha did not change significantly after excluding item 14. While item 14 could evaluate emotionalism of patients with depression, the meaning of item 14 might be interpreted in a positive way to some subjects in terms of familiar feeling or bond to others. This might have a confounding effect on validation for KDRSS, especially in oriental culture including Korea where closeness in a relationship with family or other people is regarded positively as a socially desirable value. $^{26-28}$ Therefore, item 14 should be reconsidered as an item when evaluating emotionalism related to depression.

In addition, there is a possibility that this subscale reflects personality trait of neuroticism rather than the state of depression because items 14, 16 and 23 could indicate that individuals are emotionally reactive and more vulnerable to environmental change regardless of depression. Neurotic personality trait has been reported to be associated with depressive symptoms as a risk factor for developing depression. ${ }^{29,30}$

A considerable number of FR patients (72.1\%) had at least one mild-to-severe residual subscale symptom and $9.3 \%$ of FR patients reported all 7 residual subscale symptoms. 'Lack of energy', 'increased emotionalism', and 'subjective feelings of vulnerability' subscale accounted for $45.4,40.7$, and 39.5\%, respectively. In consistent with our results, several studies have shown that anergia or fatigue is one of the most common residual symptoms of depression. ${ }^{3,31}$ Subjective feelings of vulnerability is a contrary concept of resilience defined as an individual's capacity to successfully overcome challenges and adversity in life and recover to pre-stress level of adaptation. ${ }^{32,33}$ Resilience is influenced by external environmental factors and conditions along with individual's inherent factors and traits. ${ }^{32}$ Consequently, this subscale symptom would be difficult to improve in the short term. It is likely to remain as a persistent residual symptom. The 'increased emotionalism' subscale reflects emotional expression of depression. Previous studies have suggested that post-traumatic embitterment disorder (PTED), a reactive disorder, is caused by negative life event. ${ }^{34}$ Therefore, the distinction between depression and PTED may lead to emotional upset and negative emotions remaining even after recovery from depression such as a post-traumatic stress state. These findings suggest that intense emotion may also show slow response to treatment or do not change easily, resulting in persistent residual symptoms.

The fact that patients in full remission still have various residual symptoms with high frequencies indicates that monitoring and follow-up measurement of residual symptoms is very important because these symptoms can increase the risk of relapse and affect the course of depression. In current clinical setting with limited time and resources, it is difficult to closely measure residual symptoms, suggesting that a specialized scale measuring residual symptoms such as DRSS is 
needed.

Our study had several limitations. First, considering results regarding internal consistency and factor analysis of the scale, it would be better to delete item 14 from KDRSS. Such modified KDRSS without item 14 needs to be reexamined in future studies. In addition, further studies are needed to explore the predictive value of KDRSS about recurrence of depression and its usefulness by applying this scale to various followup periods in clinical settings. Second, there are some controversies about the criteria of full remission, although the definition of remission with a HDRS-24 score of less than 10 is widely accepted. Finally, we regarded a mean KDRSS score of 1 or above on each subscale as having a residual symptom because there were no established criteria for KDRSS. If the subject had all symptoms within a subscale, it would be more obvious that they had subscale residual symptom so that we could minimize the effect of personality on items of the KDRSS.

In conclusion, this study showed excellent validity and reliability when DRSS was applied to psychiatric outpatients in Korea. Residual symptoms can be difficult to detect by examiner. They are likely to be missed without spontaneous reporting by a patient. Since the KDRSS reflects a subject's self-perception and feelings and consists of items to assess various psychological symptoms of depression, residual symptoms could be sensitively measured for patients with depression, including FR group. KDRSS is expected to contribute to identification of depressed patients with a high likelihood of recurrence. It will help establish treatment plan by sensitively detecting residual symptoms.

\section{Acknowledgments}

This research was supported by a grant of Korea Mental Health Technology R\&D Project, Ministry of Health and Welfare, Republic of Korea (grant number: HM15C1223), and partially supported by Hanlim Pharmaceuticals, Seoul, Republic of Korea.

\section{REFERENCES}

1. Kupfer DJ, Spiker DG. Refractory depression: prediction of non-response by clinical indicators. J Clin Psychiatry 1981;42:307-312.

2. McClintock SM, Husain MM, Wisniewski SR, Nierenberg AA, Stewart JW, Trivedi MH, et al. Residual symptoms in depressed outpatients who respond by $50 \%$ but do not remit to antidepressant medication. J Clin Psychopharmacol 2011;31:180-186.

3. Israel JA. The impact of residual symptoms in major depression. Pharmaceuticals (Basel) 2010;3:2426-2440.

4. Judd LL, Akiskal HS, Maser JD, Zeller PJ, Endicott J, Coryell W, et al. Major depressive disorder: a prospective study of residual subthreshold depressive symptoms as predictor of rapid relapse. J Affect Disord 1998;50:97-108.

5. Judd LL, Paulus MJ, Schettler PJ, Akiskal HS, Endicott J, Leon AC, et al. Does incomplete recovery from first lifetime major depressive episode herald a chronic course of illness? Am J Psychiatry 2000;157:15011504.

6. Keller MB. Past, present, and future directions for defining optimal treatment outcome in depression: remission and beyond. JAMA 2003;289:
3152-3160.

7. Yang H, Chuzi S, Sinicropi-Yao L, Johnson D, Chen Y, Clain A, et al. Type of residual symptom and risk of relapse during the continuation/ maintenance phase treatment of major depressive disorder with the selective serotonin reuptake inhibitor fluoxetine. Eur Arch Psychiatry Clin Neurosci 2010;260:145-150.

8. Bakish D. New standard of depression treatment: remission and full recovery. J Clin Psychiatry 2001;62(Suppl 26):5-9.

9. Boulenger JP. Residual symptoms of depression: clinical and theoretical implications. Eur Psychiatry 2004;19:209-213.

10. Cornwall PL, Scott J. Partial remission in depressive disorders. Acta Psychiatr Scand 1997;95:265-271.

11. Fava GA. Subclinical symptoms in mood disorders: pathophysiological and therapeutic implications. Psychol Med 1999;29:47-61.

12. Zimmerman M, Posternak MA, Chelminski I. Heterogeneity among depressed outpatients considered to be in remission. Compr Psychiatry 2007;48:113-117.

13. Trivedi MH. Tools and strategies for ongoing assessment of depression: a measurement-based approach to remission. J Clin Psychiatry 2009;70 (Suppl 6):26-31.

14. Bertschy G, Haffen E, Gervasoni N, Gex-Fabry M, Osiek C, Marra D, et al. Self-rated residual symptoms do not predict 1-year recurrence of depression. Eur Psychiatry 2010;25:52-57.

15. Zimmerman M, McGlinchey JB, Posternak MA, Friedman M, Attiullah N, Boerescu D. How should remission from depression be defined? The depressed patient's perspective. Am J Psychiatry 2006;163:148-150.

16. Alexopoulos GS, Katz IR, Bruce ML, Heo M, Ten Have T, Raue P, et al. Remission in depressed geriatric primary care patients: a report from the PROSPECT study. Am J Psychiatry 2005;162:718-724.

17. Montgomery SA, Asberg M. A new depression scale designed to be sensitive to change. Br J Psychiatry 1979;134:382-389.

18. Bernstein IH, Rush AJ, Stegman D, Macleod L, Witte B, Trivedi MH. A comparison of the QIDS-C16, QIDS-SR16, and the MADRS in an adult outpatient clinical sample. CNS Spectr 2010;15:458-468.

19. Carmody TJ, Rush AJ, Bernstein I, Warden D, Brannan S, Burnham D, et al. The Montgomery Asberg and the Hamilton ratings of depression: a comparison of measures. Eur Neuropsychopharmacol 2006;16:601611.

20. Uher R, Farmer A, Maier W, Rietschel M, Hauser J, Marusic A, et al. Measuring depression: comparison and integration of three scales in the GENDEP study. Psychol Med 2008;38:289-300.

21. Fava M. Somatic symptoms, depression, and antidepressant treatment. J Clin Psychiatry 2002;63:305-307.

22. Möller HJ. Methodological aspects in the assessment of severity of depression by the Hamilton Depression Scale. Eur Arch Psychiatry Clin Neurosci 2001;251(Suppl 2):13-20.

23. Suhr D. Principle Component Analysis versus Exploratory Factor Analysis. Cary, NC: SUGI 30 (SAS Users Group International); 2005.

24. Slocum-Gori SL, Zumbo BD. Assessing the unidimensionality of psychological scales: using multiple criteria from factor analysis. Soc Indic Res 2011;102:443-461.

25. Snaith RP, Harrop FM, Newby DA, Teale C. Grade scores of the Montgomery-Asberg Depression and the Clinical Anxiety Scales. Br J Psychiatry 1986;148:599-601.

26. Klopf DW. Intercultural Encounters: The Fundamentals of Intercultural Communication. Englewood, CO: Morton Publishing Company; 1998.

27. Javidi A, Javidi M. Cross-cultural analysis of interpersonal bonding: a look at East and West. Howard J Commun 1991;3:129-138.

28. Markus HR, Kitayama S. Culture and the self: implications for cognition, emotion, and motivation. Psychol Rev1991;98:224.

29. Duggan C, Sham P, Lee A, Minne C, Murray R. Neuroticism: a vulnerability marker for depression evidence from a family study. J Affect Disord 1995;35:139-143.

30. Steunenberg B, Beekman AT, Deeg DJ, Kerkhof AJ. Personality and the 
onset of depression in late life. J Affect Disord 2006;92:243-251.

31. Demyttenaere K, De Fruyt J, Stahl SM. The many faces of fatigue in major depressive disorder. Int J Neuropsychopharmacol 2005;8:93-105.

32. Jeon SW, Han C, Choi J, Pae CU, Chae JH, Ko YH, et al. Posttraumatic growth and resilience: assessment and clinical implications. J Korean Neuropsychiatr Assoc 2015;54:32-39.
33. Masten AS, Best KM, Garmezy N. Resilience and development: contributions from the study of children who overcome adversity. Dev Psychopathol 1990;2:425-444.

34. Linden M. Posttraumatic embitterment disorder. Psychother Psychosom 2003;72:195-202. 